\title{
Creation and Analysis of QR Code
}

\author{
Sudeep S. Kulkarni and Chetna Malagi
}

\begin{abstract}
QR code stands for 'Quick Response' code. It was developed by Denso Wave Corporation in Japan. A QR code is a special type of code that can encode information like numbers, letters, and Kanji characters. It is a two-dimensional code which is able to encode more information than onedimensional barcode. Also, they are fast readable codes. The emergence of smart phones increases the use of $Q R$ code because a smart phone has features of scanning and decoding a QR code. The emergence of technology in the area of mobile internet access encourages the online marketers, newspapers and magazines to use $Q R$ codes for the advertisement of their products. These codes have various significances over traditional barcodes like greater storage capacity, fast readability, 360 degree reading, small print size, error correction and support for more languages. The paper attempts to highlight the $Q R$ codes, its characteristics, creating process, encoding, decoding, significance and downsides.
\end{abstract}

Keywords--- QR Code, Barcode, Significance, Encoding, Decoding.

\section{INTRODUCTION}

$\mathrm{T}$ HE invention of barcodes was the first step towards the solution to reduce the burden of manual data entry of any goods etc. and the use of barcodes gave a relief. When the codes printed on the product were scanned by optical sensor then the price and other basic information of that product was automatically displayed.

Two dimensional barcode is developed from one dimensional barcode, and its encoding information is stored in horizontal and vertical direction. Two dimensional barcode offer many advantages: small area, high capacity, high density, error detection etc. It can be configured into a stacked or matrix format symbology. The stacked barcode is where linear bar codes are stacked one upon another and are printed in a rectangular shape to achieve the most efficient use of label area. PDF417 is a kind of stack two-dimensional barcode. The matrix barcode is that code the data based on the position of black spots within a matrix. Each black element is the same dimension and it is the position of the element that codes the data. QR Code is a typical matrix two-dimensional barcode. The barcode reader only be used to recognize the barcode, and the price of it is expensive. So mobile phones with embedded camera devices can be used to recognize the barcode.

As the use of barcodes spread, one of the prominent limitations came in the way that a barcode can only store 20

Sudeep S. Kulkarni.E-mail:Sudeepkulkarni33@gmail.com

Chetna Malagi, Student, Dept of CSE, SKSVMACET-Laxmeshwar, India. E-mail:Chetnamalagij23@gmail.com

DOI:10.9756/BIJSESC.8249 alphanumeric characters of information. But the users were demanding more storage capacity in the barcodes and then after many years of research, Denso Wave Corporation developed a new type of code which has greater storage capacity than earlier barcodes and has fast readability also. Hence the code was named as "Quick Response code" (i.e. QR code). A QR code is a special type of barcode that can encode information like numbers, letters, and Kanji characters.

\section{CHARACTERISTICS OF QR CODE}

\section{A. High Storage Capacity}

A QR code symbol can store up to 7,089 characters of information, which is a huge amount as compared to 1-D barcode.

\section{B. Encodable Character Set}

1. Numeric data (Digits 0-9)

2. Alphanumeric data (upper case letters A-Z; Digits 0 9; nine other characters: space, : $\% *+-I_{-} \$$ )

3. Kanji characters

\section{Small Printout Size}

The information in QR code is stored in both horizontal and vertical directions. Due to this feature, for the same amount of data, space acquired by QR code is one fourth times less than the space acquired by 1-D barcode.

\section{360 Degree Reading}

QR code is readable from any direction. This feature is provided by the finder patterns present at three corners of the symbol. The finder pattern helps to locate the QR code.

\section{E. Capability of Restoring and Error Correction}

If the part of code symbol is damaged or dirty, data can be recovered. The error detecting procedure can focus on the region of correct information. There are four levels of error correction of QR code that are L, M, Q and H. The level L has the weakest and level $\mathrm{H}$ has the strongest error correction capability.

\section{QR CODE PROPERTY}

A complete QR code assembly includes an encoding region and function patterns. The encoding region is used to store data, and the function patterns are used to determine the alignment of the QR code. Function patterns include position detection patterns, separators for position detection patterns, timing patterns, and alignment patterns. A two-dimensional array is used to represent the $\mathrm{QR}$ code dot matrix. The boundaries for the size $n \times n$ array are $(0$ to $(n-1), 0$ to $(n-1))$. The binary value 1 denotes the totally black module, and 0 denotes the totally white module. The QR Size denotes the number of modules in the horizontal and vertical axes of the 
QR code. Fig. 1 shows the structure of a normal QR code pattern.

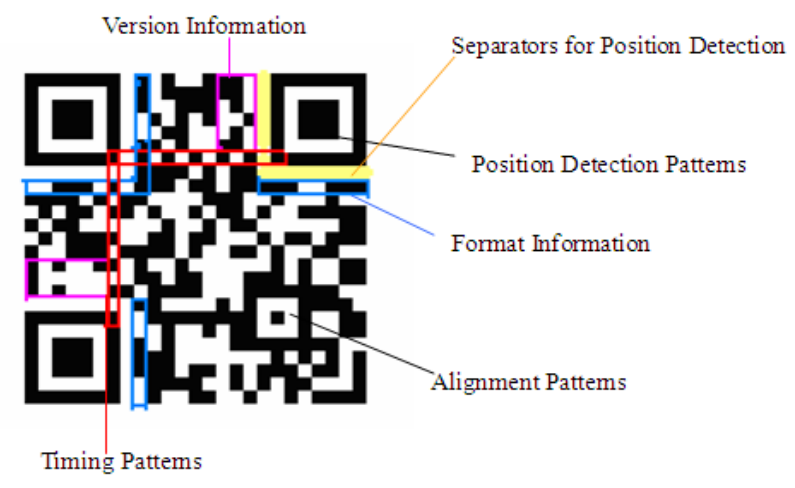

Figure 1: QR Code Structure

Position detection patterns are used to define the boundary of the entire QR code on the entire diagram. Each individual QR code has three positioning symbols that are incorporated into the $\mathrm{QR}$ code during the initial encoding and are individually distributed on the top left, bottom left, and top right of the QR code boundary. The separators for the position detection patterns are used to divide the positioning symbol and data symbol regions with widths of 1- to 0-bit and positioned individually at three locations: top left, top right, and bottom left. Timing patterns are used to determine the version information, as well as the alignment and density of the bit coordinates. Alignment patterns are used to reconstruct the corresponding bit coordinates of the concurrent image, thereby reducing the influences caused by the distorted image. The encoding region contains the data and QR Code formatting information, version information and the data, and error correction keys. The format information stores the QR code error correction level and applies encoded masking information and is a symbol required by the $\mathrm{QR}$ code decoding. The version information stores the QR codeencoded version information. The $\mathrm{QR}$ code must meet the encoded versions 7 to 40. Data and error correction code words store the encoded version information and calculated error correction key.

The error correction level indicators are shown in Table 1.

\begin{tabular}{|l|l|l|}
\hline $\begin{array}{c}\text { Error correction } \\
\text { level }\end{array}$ & $\begin{array}{c}\text { Indication bits } \\
\text { encoded value }\end{array}$ & $\begin{array}{c}\text { Number of error } \\
\text { corrections }(\%)\end{array}$ \\
\hline $\mathrm{L}$ & 00 & 7 \\
\hline $\mathrm{M}$ & 01 & 15 \\
\hline $\mathrm{Q}$ & 10 & 25 \\
\hline $\mathrm{H}$ & 11 & 30 \\
\hline
\end{tabular}

\section{General OVERVIEW OF CREATING A QR CODE}

The following pages of the tutorial will explain the $Q R$ code encoding process in detail. Here is a general overview of the process that you can read before moving on to the more detailed steps.

\section{Step 1: Data Analysis}

A QR code encodes a string of text. The QR standard has four modes for encoding text: numeric, alphanumeric, byte, and Kanji. Each mode encodes the text as a string of bits (1s and $0 \mathrm{~s}$ ), but each mode uses a different method for converting the text into bits, and each encoding method is optimized to encode the data with the shortest possible string of bits. Therefore, your first step should be to perform data analysis to determine whether your text can be encoded in numeric, alphanumeric, byte, or Kanji mode, then select the most optimal mode for your text.

\section{Step 2: Data Encoding}

Now that you have selected the appropriate encoding mode for your text, the next step is to encode the text. The data encoding section describes this process in detail for each encoding mode. The result of this step is a string of bits that is split up into data code words that are each 8 bits long.

\section{Step 3: Error Correction Coding}

As explained above, QR codes use error correction. This means that after you create the string of data bits that represent your text, you must then use those bits to generate error correction code words. QR scanners read both the data code words and the error correction code words. By comparing the two, the scanner can determine if it read the data correctly, and it can correct errors if it did not read the data correctly.

\section{Step 4: Structure Final Message}

The data and error correction code words generated in the previous steps must now be arranged in the proper order. For large QR codes, the data and error correction code words are generated in blocks, and these blocks must be interleaved according to the QR code specification.

\section{Step 5: Module Placement in Matrix}

After generating the data code words and error correction code words and arranging them in the correct order, you must place the bits in the QR code matrix. The code words are arranged in the matrix in a specific way. During this step, you will also place the patterns that are common to all QR codes, such as the boxes on the three corners.

\section{Step 6: Data Masking}

Certain patterns in the QR code matrix can make it difficult for QR code scanners to correctly read the code. To counteract this, the QR code specification defines eight mask patterns, each of which alters the QR code according to a particular pattern. You must determine which of these mask patterns results in the QR code with the fewest undesirable traits. This is done by evaluating each masked matrix based on four penalty rules. Your final QR code must use the mask pattern that resulted in the lowest penalty score.

\section{Step 7: Format and Version Information}

The final step is to add format and (if necessary) version information to the QR code by adding pixels in particular areas of the code that were left blank in previous steps. The format pixels identify the error correction level and mask pattern being used in this QR code. The version pixels encode the size of the QR matrix and are only used in larger QR codes. 


\section{A. Encoding and Decoding of the QR Code \\ a) Encoding}

The $\mathrm{QR}$ code encoding process includes the inputting of the encoded data up to the generation of the $\mathrm{QR}$ code diagram.
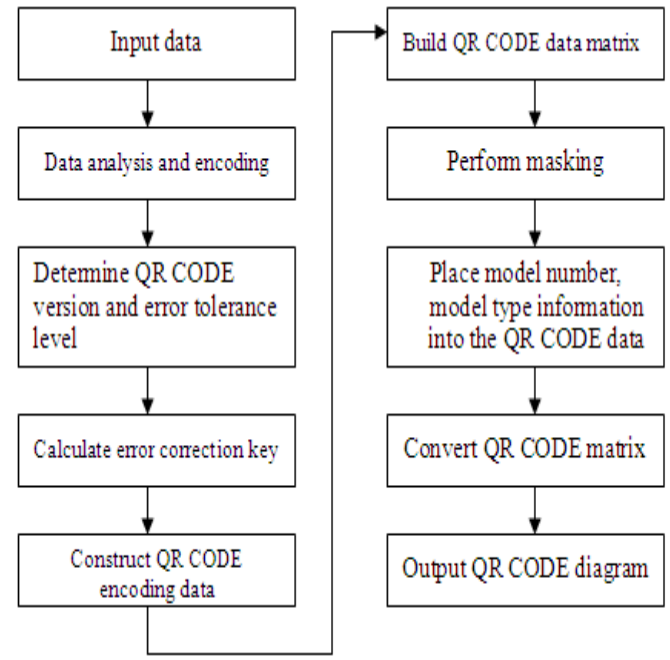

Figure 2: QR Code Encoding Flowchart

\section{b) Decoding}

The QR code decoding includes determining the region of the QR code up to obtaining the encoded character string. shows the decoding process of the standard QR code decoding.

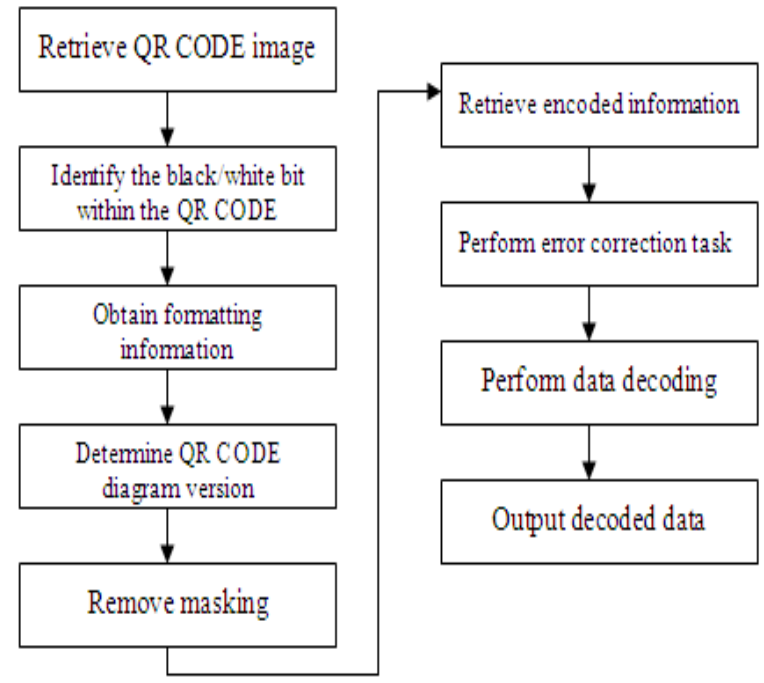

Figure 3: QR Code Decoding Flowchart

\section{SignifICANCE AND USES OF QR CODE}

QR code provides simple creation and easy access to $Q R$ code readers. Hence the QR codes are being widely used for linking to companies' website, advertising products, online menus and contest sign-up pages. QR codes printed on a building might enable the visitors to know about the history of the building or it may give the information of its architect and about how the building was built. There are many ways of using QR code. Some of them are listed below.

\section{A. On Company Business Cards}

An excellent location for QR codes is business cards. Meet somebody at any gathering, scan a code and get their contact information saved in your smart phone automatically.

\section{B. Magazine Advertisements}

The ad of a product in a magazine along with a QR code may bring the reader to the company's site and customer may read about the product and he may end up buying the product.

\section{Cafes and Restaurants}

The preferred customers of a cafe or any restaurants may be offered something free or special discounts with their next meal by using the printed $\mathrm{QR}$ code on their dining tables.

\section{Equipment Manufacturers}

The product manufacturer may add QR codes on instruction manual that can take people to the online content for helping them to use their products.

\section{E. Talking Labels}

A product label may become alive by printing QR code on it. The code may take the visitors to a video or may display some interesting photos of the product that may impress the buyers.

\section{DOWNSIDES OF QR CODE}

1. Everyone is not aware of QR codes. Consequently, not everyone who sees a QR code symbol will pull out his cell phone and take a picture of the code.

2. Also, not everyone has a camera phone and many mobile phones do not include a QR scanner.

3. Moreover, users may be directed to a web address that is not able to display properly on a mobile phone.

4. If the code printed on the product gets fully damaged during transportation then the code cannot be scanned.

\section{CONCLUSION}

In this paper, QR codes are analyzed from the perspective of their significance and uses. The working of QR code is discussed to make reader familiar with the $\mathrm{QR}$ codes. QR code can store complex information within a small code. As awareness increases about the usefulness of these codes, we can expect them to be used in more public domains.

\section{REFERENCES}

[1] International standard ISO/IEC 18004, "Information technology Automatic identification and data capture techniques Bar code symbology QR Code", Reference number, 2000.

[2] A.S. Narayanan, "QR codes and security solutions", International Journal of Computer Science and Telecommunications, Vol. 3, No. 7, Pp. 4, 2012.

[3] Q. Ji, "Exploring the concept of QR Code and the benefits of using QR Code for companies", 2014.

[4] 7 things you should know about QR Codes, EDUCAUSE Learning Initiative- advancing learning through IT innovation, retrieved from, 2016.

[5] QR_code, retrieved from, 2016.

[6] C.W. Chiang, "The Application of QR Code to Indoor Cross-Floor Automatic Route Planning", Master Find the problem of, 2009. 
[7] J.F. Weng, "The study of RSA algorithm on QR code design", Department of Computer Science and Engineering Tatung University, 2008.

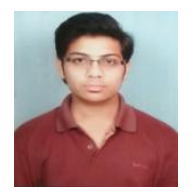

Sudeep S Kulkarni, My place of birth is Hubliand DOB is $14 / 09 / 1995$

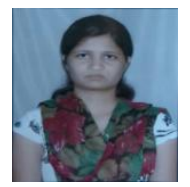

Chetna Malagi, My place of birth is Hubliand DOB is $02 / 07 / 1991$ 